\title{
Study of Thyroid Abnormalities using Scintigraphy
}

\author{
Amel Bushra. Ahmed ${ }^{1,2}$, Mohamed Yousef ${ }^{1,3}$, Salah Ali Fadhlalah ${ }^{1}$ \\ ${ }^{1}$ College of Medical Radiological Science, Sudan University of science and Technology, Sudan Khartoum \\ ${ }^{2}$ Fujairah Hospital, Fujairah, UAE \\ ${ }^{3}$ College of Batterjee Science College, Radiological Science, Jeddah, Saudi Arabia \\ Amel.Abakar@moh.gov.ae
}

\begin{abstract}
Radionuclide imaging is an integral part of functional evaluation of thyroid diseases. This study aimed to study thyroid abnormalities using scintigraphy, The study population consisted of 173(149 female and 24 male) patients with thyroid abnormalities, and referred to Nuclear Medicine, Fujairah hospital for thyroid scintigraphy during the period from Jan 10, 2016 to June 30, 2018. All patients Thyroid function test were done before coming to radiology department. The mean age of patients was 38 years.

The results of this study revealed that thyroid nuclear medicine scan findings as normal in $6.4 \%$ (16 patients) and abnormal in 91\% (157 patients), Autonomous Nodule 2 (1.1),nodular goiter- NTG , TNG 30 (17.3\%),Goiter11(6.4\%),Grave disease13(7.5\%), Multinodular goiter 43(24.8\%), Thyroiditis 20 (11), Thyroid nodule 2 (1.1), Toxic multinodular goiter 21 ,Toxic goiter 15 (8.7\%), Normal 16 (9.2\%).

This study concluded that radionuclide methods are complementary and provide information that can help in the appropriate management of various thyroid diseases.
\end{abstract}

Key words: Scintigraphy, goitre, Graves' disease, thyroid nodule

\section{Introduction}

The thyroid gland is located in the neck, superior to the trachea and between the thyroid cartilage and sternal notch. The gland is relatively small, consisting of 2 lobes, each of which is approximately $2-3 \mathrm{~cm}$ wide by $5 \mathrm{~cm}$ high. ${ }^{[1]}$.

Thyroid gland has the unique ability to take up iodine - an essential component of its hormones. The phenomenon of accumulation of iodine in the thyroid gland allowed for the use of iodine isotopes in the diagnosis of thyroid disease as early as about 70 years ago, although the mechanism of iodine uptake at the molecular level has been carefully examined until the late twentieth century. In 1939, a group of scientists from the University of Berkeley documented the uptake of radioactive iodine in human thyroid for the first time. This gave rise to first therapeutic radioiodine applications in patients with hyperthyroidism and thyroid cancer $\left.{ }^{[2,3}\right]$. Nowadays, we know that the uptake of iodine in the thyroid gland is attributed to the sodium-iodide symporter (NIS), described in 1993 by Kaminsky et al ${ }^{[4]}$. The uptake of iodine by the thyroid cells is still widely used in the evaluation of thyroid function by means of 
radioiodine uptake test and thyroid scintigraphy ${ }^{[5]}$.This study aimed to study thyroid abnormalities using scintigraphy

\section{Materials and methods}

A total of 173 patients were seen by surgeons and medical doctors as having thyroid issues, and referred to Nuclear Medicine and Radiology Department, Fujairah hospital for thyroid scintigraphy and neck ultrasound during the period from Jan. 10, 2016 to June. 30, 2018.

The thyroid scintigraphy obtained 10-20 minutes after intravenous injection of $37-111 \mathrm{MBq}$ of sodium pertechnetate Tc-99m using a LEHR (low energy high resolution collimator-equipped gammascintillation camera. All thyroid scintigraphies were interpreted by one Nuclear Medicine Physician. For ultrasound, all patients were scanned supine with their necks hyperextended using a 7.5-10-MHz transducer. All patients underwent neck ultrasound by one expert radiologist. The data analyzed using the SPSS program.

\section{Nuclear medicine examination}

Technetium $99 \mathrm{~m}$ pertechnetate ( $99 \mathrm{mTcO} 4-)$ is a used radioactive label for thyroid scanning. Injection Technique a fine Butterfly needle (gauge 23-25 according to patient's age) is recommended. Technetium-99m when used as a radioactive tracer can be detected in the body by medical equipment (gamma cameras). It is well suited to the role because it emits readily detectable $140 \mathrm{keV}$ gamma rays (these are about the same wavelength as emitted by conventional $X$-ray diagnostic equipment), and its half-life for gamma emission is 6.0058 hours (meaning $93.7 \%$ of it decays to 99Tc in 24 hours). The "short" physical half-life of the isotope and its biological half-life of 1 day (in terms of human activity and metabolism) allows for scanning procedures which collect data rapidly, but keep total patient radiation exposure low. The same characteristics make the isotope suitable only for diagnostic but never therapeutic use.

\section{Data Collection and Analysis}

Data will be collected in tabulated database sheet and will be analyzed by SPSS. The data included the age , gender, Us finding and Nuclear medicine scintigraphy findings

\section{Results}

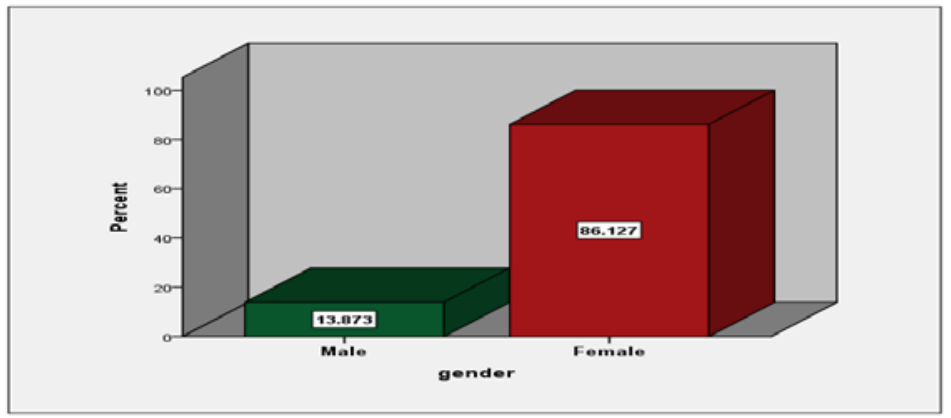

Figure 1 shows gender of the patients 


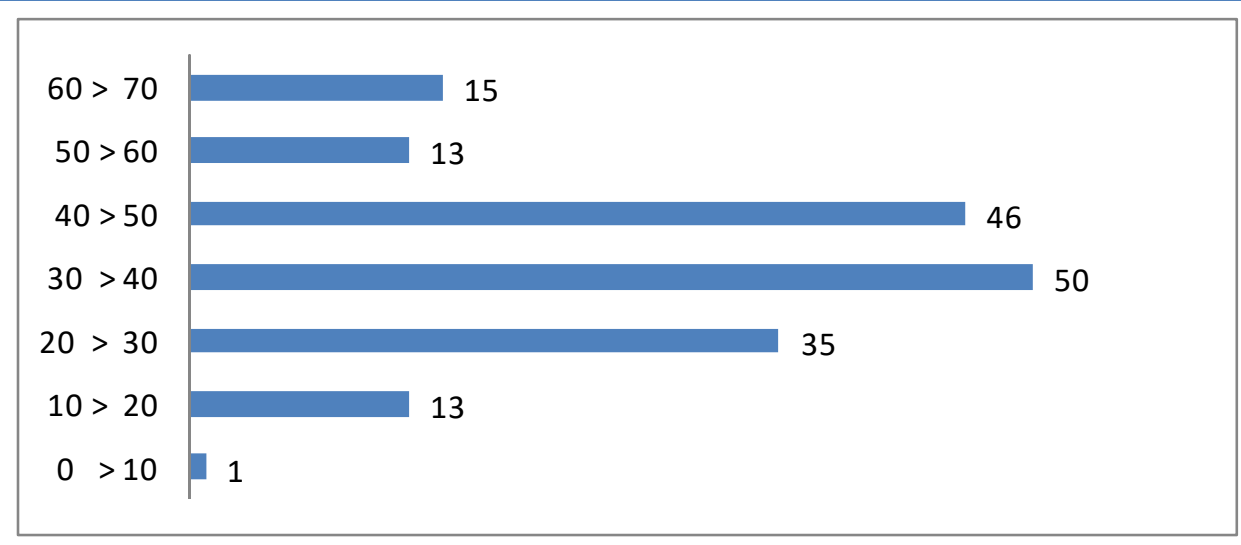

Figure $\mathbf{2}$ shows patient's ages

Table 1 shows NM findings

\begin{tabular}{|l|c|}
\hline Thyroid disorders & Frequency \\
\hline Autonomous Nodule & $2(1.1)$ \\
\hline nodular goiter- NTG, TNG & $30(17.3 \%)$ \\
\hline Goiter & $11(6.4 \%)$ \\
\hline Grave disease & $13(7.5 \%)$ \\
\hline Multinodular goiter & $43(24.8 \%)$ \\
\hline Thyroiditis & $20(11)$ \\
\hline Thyroid nodule & $2(1.1)$ \\
\hline Toxic multinodular goiter & 21 \\
\hline Toxic goiter & $15(8.7 \%)$ \\
\hline Normal & $16(9.2 \%)$ \\
\hline total & 173 \\
\hline
\end{tabular}

Table 2 shows Nodules sites

\begin{tabular}{|l|c|}
\hline Nodule site & Frequency \\
\hline Lt lobe & 63 \\
\hline Rt lobe & 80 \\
\hline isthmus & 19 \\
\hline Total & 162 \\
\hline
\end{tabular}

Table 3 shows Thyroid size

\begin{tabular}{|l|l|}
\hline size & Frequency \\
\hline Normal & $49(28 \%)$ \\
\hline Enlarge & $124(72 \%)$ \\
\hline Total & $173(100 \%)$ \\
\hline
\end{tabular}




\section{Discussion}

Nuclear scintigraphy is commonly used for evaluation of physiologic thyroid function and for identification of metabolically active and inactive nodules.

Nuclear medicine thyroid scan was performed by an Bright View gamma camera. Nuclear medicine classification based on distribution of the radioisotopes through the thyroid. Features were performed by one experienced nuclear medicine technologist. Size was measured as the whole diameter. The features included, hemogeniousity, uptake, count pear pixel, and area.

The study population consisted of 173patients were seen by surgeons and medical doctors as having thyroid issues, and referred to Nuclear Medicine and Radiology Department, Fujairah hospital for thyroid scintigraphy and neck ultrasound during the period from Jan. 10, 2016 to June. 30, 2018.All patients Thyroid function test were done before coming to radiology department. So all results were comparing with the TFT results.

nuclear medicine thyroid scintigraphy and demographic data are presented in Tables and figures for this study revealed that, among this 173 patients $86 \%$ were female(149) and $14 \%$ were male (24), fig(1).The mean age of patients was 38 years, thyroid nuclear medicine scan were reported as normal in $6.4 \%$ (16 patients) and abnormal in 91\% (157 patients) .

In this study table (1) shows that nuclear medicine is able to detect multi nodules in 43 patients. and if multi nodules are toxic, nuclear medicine is better in detecting it (21 patients) In diagnosis of Autonomous nodule nuclear scan was able to detect it In graves' disease nuclear medicine (13 patients) $7.5 \%$ in detecting it. For thyroiditis nuclear medicine, (25 patients) $14.4 \%$ to (20 patients) $11 \%$. However in diagnosis of toxic goiter nuclear medicine thyroid scan was better than ultrasound, (15 patients) $8.7 \%$

Table (2) shows the nodule sites in the Rt lobe, Lt lobe and isthmus, 80, 63, 19 in the thyroid nuclear medicine scan.

Table (3) shows that the thyroid gland size if its enlarge or not. In nuclear medicine thyroid scan it was enlarge in (124 patients) $72 \%$ of patients and normal in (49 patients) $28 \%$ of all cases.

In spite of some limitations of this, ultrasound examination plays an important role for patients in diagnosis of thyroid abnormalities, especially in thyroid nodules.

\section{Conclusion}

This study concluded that radionuclide methods can be able to provide information that can help in the appropriate management of various thyroid diseases.

\section{REFERENCES}

[1] Drew HH, LaFrance ND, Chen JS. Thyroid imaging studies. J Nucl Med Technol. 1987;15:79-87

[2] Becker DV, Sawin CT. Radioiodine and thyroid disease: the beginning. Semin Nucl Med 1996; 26: 155-164. 
Amel Bushra. Ahmed, Mohamed Yousef, Salah Ali Fadhlalah; Study of Thyroid Abnormalities using Scintigraphy. Journal of Biomedical Engineering and Medical Imaging, Volume 5, No 5 October (2018), pp 1-5

[3] Keston AS, Ball RP, Frantz VK, Palmer WW. Storage of radioactive iodine in a metastasis from thyroid carcinoma. Science 1942; 2466: 362-363.

[4] Kaminsky SM, Levy O, Salvador C, Dai G, Carrasco N. The Na+/Isymporter of the thyroid gland. Soc Gen Physiol Ser 1993; 48: 251-262.

[5] Chung JK. Sodium iodide symporter: its role in nuclear medicine. J Nucl Med 2002; 43: 11881200. 